\title{
ON THE DERIVATIVE OF A UNIVALENT FUNCTION
}

\section{A. J. LOHWATER AND GEORGE PIRANIAN}

Various results are known concerning the rate of growth of the derivative of a function $f(z)$, analytic and univalent in the circle $|z|<1$; among the simplest of these results we mention Koebe's Verzerrungsatz (cf. $[4$, p. 86$]$ ), according to which $\left|f^{\prime}(z)\right|$ $=O\left[(1-|z|)^{-8}\right]$ in $|z|<1$. However, as rapid a rate of growth as is permitted by this theorem can be attained only on a relatively small set of radii; indeed, Seidel and Walsh [5] have shown that for all $e^{i \theta}$ on $|z|=1$, except for a possible set of linear measure zero,

$$
\lim _{r \rightarrow 1}(1-r)^{1 / 2}\left|f^{\prime}\left(r e^{i \theta}\right)\right|=0 .
$$

It is a simple consequence of (1) that almost every radius of $|z|<1$ is mapped by $w=f(z)$ onto a rectifiable arc terminating at a frontier point of that region $G$ in the $w$-plane which is the image of $|z|<1$ under $f(z)$.

Recently there has been some success in cutting down the size of certain exceptional sets associated with a univalent function and its derivative. In particular, we cite Beurling's result [1] that the set of points $e^{i \theta}$ for which the integral $\int_{0}^{1}\left|f^{\prime}\left(r e^{i \theta}\right)\right| d r$ is infinite (and consequently, the set of points for which $\lim _{r \rightarrow 1} f\left(r e^{i \theta}\right)$ does not exist) is a set of logarithmic capacity zero in the sense of Frostman [3]. From Beurling's result it follows immediately that the radii drawn to all points $e^{i \theta}$ of $|z|=1$, except possibly for a set of logarithmic capacity zero, are mapped by $w=f(z)$ onto rectifiable arcs terminating at frontier points of the region $G$, a fact which has been pointed out by Dufresnoy [2] and Tsuji [6].

It is the object of this note to show that the distortion theorem of Seidel and Walsh mentioned above cannot be subjected to the analogous improvement, i.e., that the set of directions for which $\lim _{r \rightarrow 1}(1-r)^{1 / 2}\left|f^{\prime}\left(r e^{i \theta}\right)\right|>0$ is not necessarily of capacity zero. Whether (1) can be improved (with an exceptional set of linear measure zero) by replacing $(1-r)^{1 / 2}$ with a function of $r$ which tends to zero more slowly than $(1-r)^{1 / 2}$ is still not known.

We begin by proving a lemma on the rate of growth of a class of harmonic functions.

Presented to the Society, November 29, 1952; received by the editors September 17, 1952 and, in revised form, October 30, 1952. 
LEMMA. Let $\mu(t)$ be a monotonic, nondecreasing function of bounded variation in $-\pi \leqq t \leqq \pi$, and let $u(r, \theta)$ be the harmonic function

$$
u(r, \theta)=\frac{1}{2 \pi} \int_{-\pi}^{\pi} \frac{1-r^{2}}{1+r^{2}-2 r \cos (\theta-t)} d \mu(t), \quad r<1 .
$$

If $t_{0}$ is a point of the interval $[-\pi, \pi]$ for which

$$
\lim _{h \rightarrow 0} \frac{\mu\left(t_{0}+h\right)-\mu\left(t_{0}-h\right)}{h^{\alpha}}=\infty, \quad 0<h, 0<\alpha \leqq 1,
$$

then

$$
\lim _{r \rightarrow 1}(1-r)^{1-\alpha} u\left(r, t_{0}\right)=\infty .
$$

It may be assumed in the proof, without loss of generality, that $t_{0}=0$. For an arbitrarily large positive number $M$, condition (3) permits us to choose $r_{0}<1$ so that $(\mu(h)-\mu(-h)) / h^{\alpha}>M$ whenever $h<1-r_{0}$. The integral (2) may be estimated as follows for $\theta=t_{0}=0$ :

$$
\begin{aligned}
u(r, 0) & =\frac{1}{2 \pi} \int_{-\pi}^{\pi} \frac{1-r^{2}}{1+r^{2}-2 r \cos t} d \mu(t) \\
& \geqq \frac{1}{2 \pi} \int_{-(1-r)}^{1-r} \frac{1-r^{2}}{1+r^{2}-2 r \cos t} d \mu(t) .
\end{aligned}
$$

For $|t| \leqq \pi$

$$
\begin{aligned}
\frac{1-r^{2}}{1+r^{2}-2 r \cos t} & =\frac{1-r^{2}}{1+r^{2}-2 r\left(1-t^{2} / 2 !+t^{4} / 4 !+\cdots\right)} \\
& \geqq \frac{1-r^{2}}{(1-r)^{2}+r t^{2}},
\end{aligned}
$$

so that it follows from $|t| \leqq 1-r$ that

$$
\begin{aligned}
u(r, 0) & \geqq \frac{1}{2 \pi} \int_{-(1-r)}^{1-r} \frac{1-r^{2}}{(1-r)^{2}(1+r)} d \mu(t) \\
& =\frac{1}{2 \pi(1-r)^{1-\alpha}} \cdot \frac{\mu(1-r)-\mu(r-1)}{(1-r)^{\alpha}} .
\end{aligned}
$$

Hence $(1-r)^{1-\alpha} u(r, 0) \geqq M / 2 \pi$, which proves the lemma. We remark that the proof depends strongly on the radial approach, and that the lemma need not be true for approach in the "wide sense."

THEOREM. There exists a function $f(z)$, analytic and univalent in 
$|z|<1$, and $a$ set $E$ on $|z|=1$ of positive logarithmic capacity, such that $\lim _{r \rightarrow 1}(1-r)^{1 / 2}\left|f^{\prime}\left(r e^{i \theta}\right)\right|=\infty$ whenever $e^{i \theta}$. belongs to $E$.

Consider the perfect nowhere dense set $E$ lying in the interval $[0,1]$ and constructed like the classical middle-thirds Cantor set, except that the intervals deleted at the $k$ th stage constitute the middle three-fourths rather than the middle thirds. Since a constant multiple (namely, three-fourths) is removed at each stage of the construction of $E$, it follows from [4, pp. 119 and 149] that $E$ has positive capacity. We form the monotone nondecreasing singular function $\mu(t), 0 \leqq t \leqq 1$, associated with $E$, and we proceed to show that, for each $t$ in $E$,

$$
\lim _{h \rightarrow 0} \frac{\mu(t+h)-\mu(t-h)}{h^{1 / 2}}=\infty .
$$

Let $t$ be any point in $E$ (for the sake of convenience, we assume $t$ to be different from 0 or 1 ), and let $h$ be any positive number such that $0 \leqq t-h<t+h \leqq 1$. Let $k$ denote that stage in the construction of $E$ for which at least one of the deleted intervals $I_{k}$ lies completely in $(t-h, t+h)$, while no interval $I_{k-p}(p>0)$ lies completely in $(t-h$, $t+h)$. The length of $I_{k}$ is $(3 / 4) 8^{1-k}$. We then have the inequality $h<7 / 8^{k-1}<8^{2-k}$. On the other hand, at least one of the points $t \pm h$ lies in an interval $I_{k-p}(p>0)$, so that we have the estimate $\mu(t+h)$ $-\mu(t-h) \geqq 1 / 2^{k}$, and the assertion follows.

If $\mu(t)$ is extended to the interval $-\pi \leqq t \leqq \pi$ by defining $\mu(t)$ to be 0 if $-\pi \leqq t \leqq 0$ and 1 if $1 \leqq t \leqq \pi$, and if $u(r, \theta)$ is the harmonic function (2) corresponding to $\mu(t)$, then it follows from the lemma, with $\alpha=1 / 2$, that $\lim _{r \rightarrow 1}(1-r)^{1 / 2} u(r, \theta)$ is infinite on the set $E$. To complete the proof of the theorem, we consider the function

$$
\Phi(z)=\frac{1}{2 \pi} \int_{-\pi}^{\pi} \frac{e^{i t}+z}{e^{i t}-z} d \mu(t)
$$

which is analytic in $|z|<1$, and whose real part is the positive harmonic function $u(r, \theta)$. Since $u(r, \theta) \leqq\left|\Phi\left(r e^{i \theta}\right)\right|$, it follows that $\lim _{r \rightarrow 1}(1-r)^{1 / 2}\left|\Phi\left(r e^{i \theta}\right)\right|$ is infinite on the set $E$. Next, it is a simple matter to show that the function $f(z)$ defined by

$$
f(z)=\int_{0}^{z} \Phi(\zeta) d \zeta
$$

is univalent in $|z|<1$. Indeed, it follows from (5) that

$$
f\left(z_{2}\right)-f\left(z_{1}\right)=\int_{z_{1}}^{z_{2}} \Phi(\zeta) d \zeta
$$


so that, if $z_{1} \neq z_{2}$, and if the path of integration is taken to be the line segment $\zeta=\tau\left(z_{2}-z_{1}\right)+z_{1}, 0 \leqq \tau \leqq 1$, we have

$$
\frac{f\left(z_{2}\right)-f\left(z_{1}\right)}{z_{2}-z_{1}}=\int_{0}^{1} \Phi\left[\tau\left(z_{2}-z_{1}\right)+z_{1}\right] d \tau .
$$

By taking the real parts of both sides and observing that $\operatorname{Re} \Phi(z)$ $=u(r, \theta)>0$, we see that the left-hand side can never vanish. Hence the function $f(z)$ defined by $(5)$ is univalent in $|z|<1$. Hence, for the univalent function $f(z)$, we must have $\lim _{r \rightarrow 1}(1-r)^{1 / 2}\left|f^{\prime}\left(r e^{i \theta}\right)\right|=\infty$ on the set $E$. This proves the theorem.

We remark that, if $0<\alpha \leqq 1$, it is possible to construct a singular function $\mu(t)$ such that (3) holds on a set of positive capacity. Applying the method of the last paragraph, we obtain the following more general result: For every $\beta$ with $0 \leqq \beta<1$ there exists a set $E_{\beta}$ on $|z|=1$ of positive logarithmic capacity and a function $f(z)$, analytic and univalent in $|z|<1$, such that $\lim _{r \rightarrow 1}(1-r)^{\beta}\left|f^{\prime}\left(r e^{i \theta}\right)\right|$ is infinite whenever $e^{i \theta}$ belongs to $E_{\beta}$.

\section{BibLIOGRAPHY}

1. A. Beurling, Ensembles exceptionnels, Acta Math. vol. 72 (1940) pp. 1-13.

2. J. Dufresnoy, Sur la correspondance des frontières dans la representation conforme, C.R. Acad. Sci. Paris vol. 220 (1945) pp. 189-190.

3. O. Frostman, Potential d'Equilibre et capacite des ensembles avec quelques applications a la theorie des fonctions, Meddelanden från Lunds Universitets Matematiska Seminarium vol. 3 (1935) pp. 1-115.

4. R. Nevanlinna, Eindeutige analytische Funktionen, Berlin, 1936.

5. W. Seidel and J. L. Walsh, On the derivatives of functions analytic in the unit circle and their radii of univalence and of p-valence, Trans. Amer. Math. Soc. vol. 52 (1942) pp. 128-216.

6. M. Tsuji, Beurling's theorem on exceptional sets, Tohoku Math. J. vol. 2 (1950) pp. 113-125.

ThE UNIVERSity OF Michigan 